\title{
Effect of depolarization and coercivity on actuation strains due to domain switching in barium titanate
}

\author{
Y. C. Shu ${ }^{\text {a) }}$ and J. H. Yen \\ Institute of Applied Mechanics, National Taiwan University, Taipei 106, Taiwan, Republic of China \\ J. Shieh and J. H. Yeh \\ Department of Materials Science and Engineering, National Taiwan University, Taipei 106, Taiwan, \\ Republic of China
}

(Received 13 February 2007; accepted 24 March 2007; published online 23 April 2007)

\begin{abstract}
Large electrostrictive actuation in ferroelectric single crystals can be achieved through non- $180^{\circ}$ domain switching using various biasing fields and loads. The theoretical maximum actuation strain such as $1.1 \%$ for barium titanate crystals, however, has not been observed yet. In this letter, the authors propose a possible mechanism accounting for the depolarization effect to explain the significant strain reduction observed in their recent experiment. They find that a low-energy path requires the switching of $90^{\circ}$ domains together with that of in-plane alternating layers of $180^{\circ}$ domains formed to reduce the depolarization energy. Therefore, reduction in strain is significant for crystals with large $180^{\circ}$ coercivities. The result is consistent with their recent experimental observations. () 2007 American Institute of Physics. [DOI: 10.1063/1.2731315]
\end{abstract}

The interest in large strain actuation has motivated many experimental efforts using ferroelectric single crystals to achieve this goal. A variety of attempts has been carried out by inducing large changes in the polarization orientation either via phase transition or via domain switching. Park and Shrout utilized the phase transition to obtain "ultrahigh strain" in a single crystal relaxor ferroelectric PZN-PT (a solid solution of lead zirconate niobate and lead titanate) which possesses an electrostrictive strain up to $1.7 \%$ at around $10 \mathrm{MV} \mathrm{m}^{-1}$. $^{1}$ Ren demonstrated that the symmetryconforming property of point defects can give rise to strain up to $0.75 \%$ at $0.2 \mathrm{MV} \mathrm{m}^{-1}$ in thermally aged barium titanate $\left(\mathrm{BaTiO}_{3}\right)$ crystals. $^{2}$ Burcsu et al. examined the $90^{\circ}-$ switching-induced strain of $\mathrm{BaTiO}_{3}$ crystals under a combined electrical and mechanical loading, and observed nearly $0.8 \%$ strain at $1.78 \mathrm{MPa}$ compressive stress together with $1 \mathrm{MV} \mathrm{m}^{-1}$ electric field. ${ }^{3,4}$ However, the maximum actuation strain allowed by crystallography, such as $1.1 \%$ predicted for $\mathrm{BaTiO}_{3}$ single crystals, ${ }^{5}$ has not been observed yet. This has motivated a number of research efforts to study the switching mechanism experimentally and theoretically. ${ }^{6-9}$ In particular, since large strain behavior caused by domain switching is typically induced through angles other than $180^{\circ}$, it is therefore essential to investigate factors which might influence the "effectiveness" of non- $180^{\circ}$ switchings. Shilo et al. have recently proposed a switching model to explain the strain reduction found in the experimental work of Burcsu et al. ${ }^{10}$ Their model has assumed the existence of friction between the ferroelectric crystal and the loading device, showing that the strain is reduced due to this factor. The current investigation, on the other hand, focuses on another possible mechanism accounting for the depolarization effect for the switching hardening.

Consider a ferroelectric single crystal described by two state variables: strain $\varepsilon$ and polarization $\mathbf{p}$. The reduction in crystal symmetry from the paraelectric to the ferroelectric

\footnotetext{
${ }^{\text {a) }}$ Author to whom correspondence should be addressed; electronic mail:
} yichung@spring.iam.ntu.edu.tw phase implies that the ferroelectric crystal has several ground states (variants) in the strain-polarization space. For $\mathrm{BaTiO}_{3}$ in the tetragonal phase, there exist six possible variants with the polarization vector in one of the six pseudocubic $\langle 100\rangle$ directions, and the different variants can coexist as "domains" in very intricate and characteristic patterns: the underlying distortion must be continuous and the interface must be uncharged. A detailed classification of domain patterns in ferroelectric crystals has been explored by Shu and Bhattacharya, ${ }^{5}$ and their analysis suggests that there exists a low-energy path for switching from one polarization to another by a combined electrical and mechanical loading. Indeed, for a flat-plate configuration with electrodes on the top and bottom surfaces, the potential energy per unit volume of the system in the two-dimensional (2D) version of the framework $^{5}$ can be written as

$$
\langle I\rangle=-E_{3}^{*}\left\langle p_{3}\right\rangle-\sigma_{33}^{*}\left\langle\varepsilon_{33}\right\rangle+\frac{C}{2 \epsilon_{0}}\left\langle p_{1}\right\rangle^{2},
$$

where $\langle\cdots\rangle$ stands for the volume average, $E_{3}^{*}$ and $\sigma_{33}^{*}$ are the applied electric field and stress, $\epsilon_{0}$ the permittivity of free space, and $C$ is a constant related to the geometry of the crystal. Several comments for Eq. (1) are made here. First, the last term of Eq. (1) is the contribution of the depolarization energy. For a rectangular geometry with thickness $H$ and length $\quad L, \quad C \approx 8(H / L) \sum_{n=1}^{\infty}\left[1-(-1)^{n}\right] / n^{3} \pi^{3}\left(1-e^{-n \pi(L / H)}\right)$. Therefore, unless the ratio $H / L$ is extremely small, the effect of depolarization energy may not be neglected. Second, Eq. (1) has no contribution from the depolarization in the interior since the domain patterns are assumed to be compatible. ${ }^{11}$ Third, while there are various candidates of domains which can be chosen for Eq. (1), an important one is a multirank laminate. For example, rank-1 lamellar and rank-2 banded domains are commonly observed patterns in $\mathrm{BaTiO}_{3}{ }^{12,13}$ Further, it has been shown that, given an arbitrary average state of strain and polarization, a minimum energy configuration of domains can be constructed through multirank lamination. ${ }^{14-16}$ Based on this result, the lowest rank laminate satisfying the above property in a $2 \mathrm{D}$ tetragonal $\mathrm{BaTiO}_{3}$ 


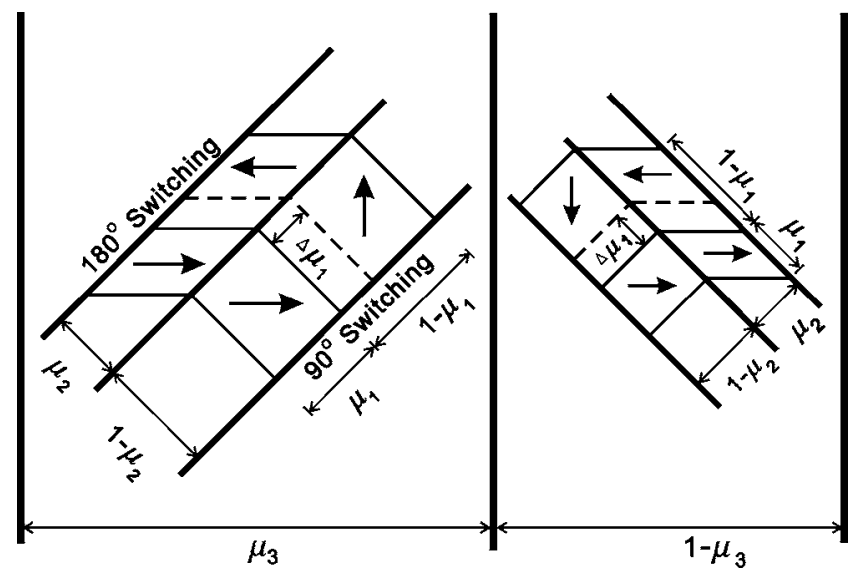

FIG. 1. Compatible domain pattern using rank-3 lamination in a $\mathrm{BaTiO}_{3}$ single crystal. Notice that the change in the local volume fraction $\mu_{1}$ induces two types of domain wall movements.

crystal is a three-rank compatible domain pattern as illustrated in Fig. 1. The polarization and strain averages of this pattern are given by

$$
\begin{aligned}
& \left\langle p_{1}\right\rangle=\left[\mu_{1}-\left(1-\mu_{1}\right) \mu_{2}\right] P_{s}, \\
& \left\langle p_{3}\right\rangle=\left(1-\mu_{1}\right)\left(1-\mu_{2}\right)\left(2 \mu_{3}-1\right) P_{s}, \\
& \left\langle\varepsilon_{33}\right\rangle=\eta_{1}+\left(\eta_{2}-\eta_{1}\right)\left[\mu_{1}+\left(1-\mu_{1}\right) \mu_{2}\right],
\end{aligned}
$$

where $\eta_{1}=0.67 \%, \eta_{2}=-0.42 \%$, and $P_{s}=0.26 \mathrm{C} \mathrm{m}^{-2}$ are measured parameters for spontaneous strain and polarization, ${ }^{17}$ and $\mu_{1}, \mu_{2}$, and $\mu_{3}$ denote the local volume fractions appearing in the first, second, and third ranks, respectively.

The reduction in potential energy provides the (thermodynamic) driving force for the domain evolution. However, switching is resisted by the dissipative motion of domain walls, ${ }^{7}$ and as a result, domain switching occurs when

$$
\frac{\partial}{\partial \mu_{i}}\langle I\rangle(\boldsymbol{\mu}) d \mu_{i}+G_{i}^{c}(\boldsymbol{\mu})\left|d \mu_{i}\right| \leqslant 0,
$$

where $G_{i}^{c}(\boldsymbol{\mu})$ is the critical dissipative energy density and is explained as follows. Assume that volume fractions $\mu_{2}$ and $\mu_{3}$ shown in Fig. 1 are fixed. The change in $\mu_{1}$ will involve two types of domain wall movements: $180^{\circ}$ wall motion in the region with local volume fraction $\mu_{2}$ and $90^{\circ}$ wall motion in another region with local volume fraction $\left(1-\mu_{2}\right)$. Let

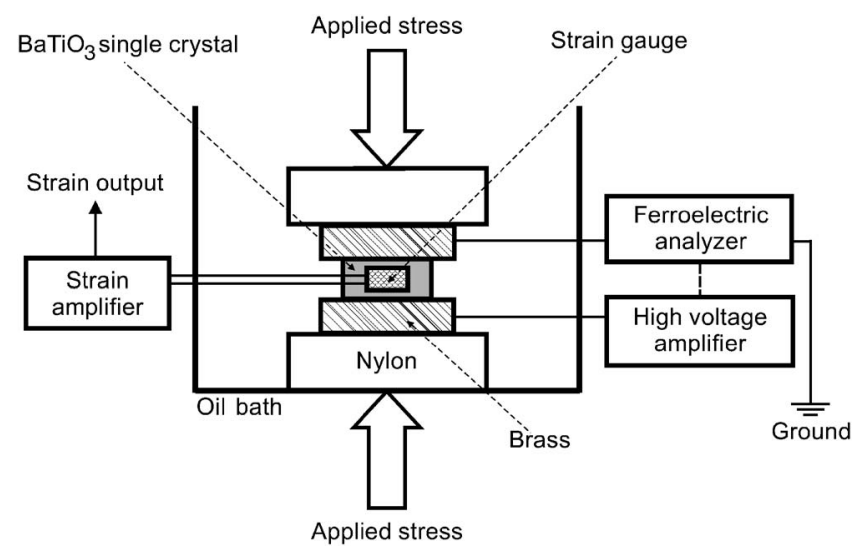

FIG. 2. Schematic of the combined electromechanical loading apparatus. Downloaded 10 Dec 2008 to 140.112 113.225. Redistribution subject

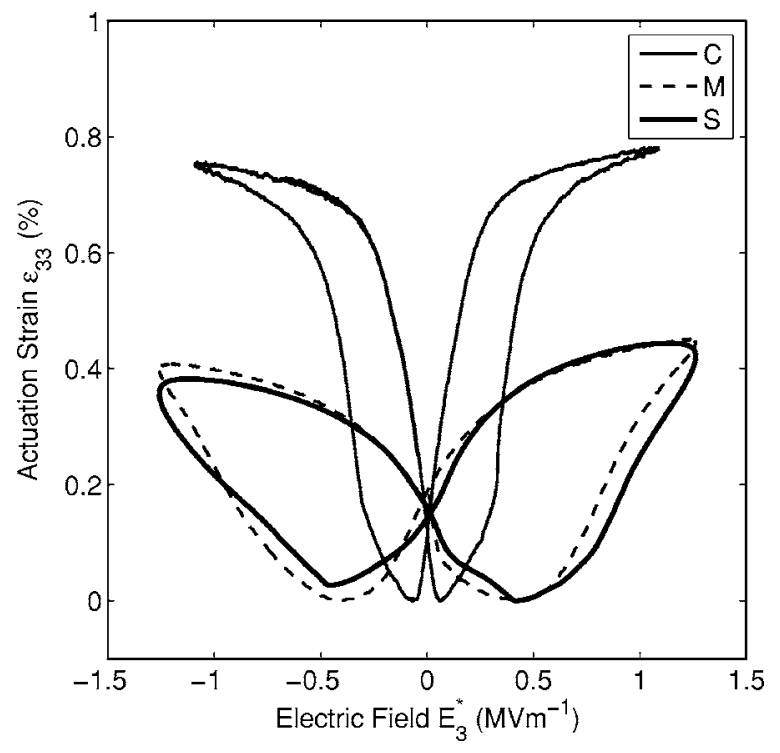

FIG. 3. Experimental observations of maximum actuation strains from three different $\mathrm{BaTiO}_{3}$ single crystals (labeled $\mathrm{C}, \mathrm{M}$, and $\mathrm{S}$ ) under combined electrical and mechanical loadings.

$E_{180^{\circ}}^{c}$ and $E_{90^{\circ}}^{c}$ denote the coercive electric fields necessary to move $180^{\circ}$ and $90^{\circ}$ domain walls in tetragonal crystals, respectively. The dissipation energy density due to the change in $\mu_{1}$ is therefore

$$
\begin{aligned}
G_{1}^{c}(\boldsymbol{\mu}) & =\left(\mathbf{E}_{180^{\circ}}^{c} \cdot \Delta \mathbf{p}\right) \mu_{2}+\left(\mathbf{E}_{90^{\circ}}^{c} \cdot \Delta \mathbf{p}\right)\left(1-\mu_{2}\right) \\
& =2 P_{s} E_{180^{\circ}}^{c} \mu_{2}+P_{s} E_{90^{\circ}}^{c}\left(1-\mu_{2}\right) .
\end{aligned}
$$

Other explicit expressions of $G_{2}^{c}(\boldsymbol{\mu})$ and $G_{3}^{c}(\boldsymbol{\mu})$ can also be derived in a similar way with a different competition between $E_{180^{\circ}}^{c}$ and $E_{90^{\circ} \text {. }}^{c}$

From Eq. (4), the switching starts when the applied electric field and stress reach the values determined by

$$
-P_{s} E_{3}^{*}+\left(\eta_{2}-\eta_{1}\right) \sigma_{33}^{*}+\frac{C}{\epsilon_{0}} P_{s}\left\langle p_{1}\right\rangle=P_{s} E_{90^{\circ}}^{c} .
$$

Notice that if the depolarization effect is not taken into account, i.e., $C=0$ in Eq. (6), the $90^{\circ}$ switching process would

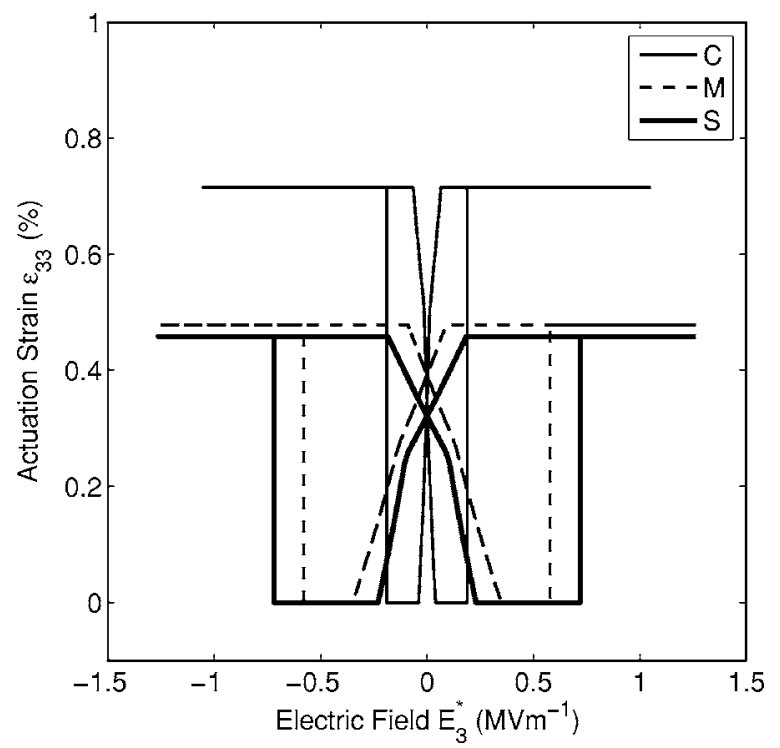

FIG. 4. Simulations of actuation strains in comparison with experimental observations in Fig. 3.

to AIP license or copyright; see http://apl.aip.org/apl/copyright.jsp 
be completed if the electromechanical loading is balanced by the coercive field $P_{s} E_{90^{\circ}}^{c}$, and therefore, the actuation strain would achieve its maximum value $\eta_{1}-\eta_{2}$. If, however, $C \neq 0$ in Eq. (6), the switching depends on $\left\langle p_{1}\right\rangle$ which is related to the local volume fractions $\mu_{1}$ and $\mu_{2}$ by Eq. (2). The amount of $\mu_{2}$ can be determined by

$$
-P_{s} E_{3}^{*}+\left(\eta_{2}-\eta_{1}\right) \sigma_{33}^{*}=P_{s} E_{90^{\circ}}^{c}+P_{s} E_{180^{\circ}}^{c} \mu_{2},
$$

and $\mu_{1}$ is solved from Eq. (6). Physically, as illustrated in Fig. 1, the in-plane alternating layers of $180^{\circ}$ domains are formed to reduce the depolarization energy. The compatibility in domain structure requires that the change in $\mu_{1}$ induces both the $90^{\circ}$ and $180^{\circ}$ switchings. Therefore, large coercive field $E_{180^{\circ}}^{c}$ implies a significant amount of energy dissipated in moving these in-plane layered domains, which in turn is not favorable for the $90^{\circ}$ switching process. Indeed, from Eq. (7), larger $E_{180^{\circ}}^{c}$ gives rise to smaller $\mu_{2}$ at the same level of applied field and stress, resulting in smaller strain in actuation.

The theoretical framework described above is developed to explain a series of experiments for measuring actuation strains of $\mathrm{BaTiO}_{3}$ single crystals under combined electrical and mechanical loadings. (001)-oriented cuboidal $\mathrm{BaTiO}_{3}$ single crystals measuring $5 \times 5 \times 2 \mathrm{~mm}^{3}$ were obtained from MTI Corp., CA (labeled M) and Superconix Inc., MN (labeled S). Conductive silver paint was used to produce electrodes on the $5 \times 5 \mathrm{~mm}^{2}$ faces. A loading fixture shown in Fig. 2 was developed for the simultaneous application of compressive stress and electric field. The mechanical and electrical loadings were supplied by a universal test frame and a high-voltage amplifier, respectively. Miniature strain gauges were attached onto the $5 \times 2 \mathrm{~mm}^{2}$ faces of the crystal to measure strain changes in the loading direction. Besides the strain measurements, the two input parameters required for the theoretical framework, $E_{180^{\circ}}^{c}$ and $E_{90^{\circ}}^{c}$ of the single crystal, were obtained, respectively, from the polarization hysteresis at zero compressive stress and from the in situ domain observation using crossed polarizers under an arrangement where the applied electric field was perpendicular to the poling direction of the polished crystal.

Upon the application of combined loadings, the maximum actuation strains produced by different $\mathrm{BaTiO}_{3}$ single crystals are shown in Fig. 3 in the form of largest strain hysteresis (i.e., butterfly) curves. Figure 3 also displays the experimental strain data of the single crystal of Burcsu et al. (labeled C). ${ }^{4}$ It is found from Fig. 3 that crystals $\mathrm{M}$ and $\mathrm{S}$ exhibit more squashed and broadened butterfly curves and significantly lower strain outputs in comparison with crystal C. Indeed, the measured strains for crystals $\mathrm{M}$ and $\mathrm{S}$ are about $0.4 \%$ while crystal $\mathrm{C}$ produced a strain close to $0.8 \%$. To explain this, the butterfly loops are simulated using Eq. (4) as well as the coercive fields measured independently from Fig. 3. The $E_{180^{\circ}}^{c}$ is about $0.2-0.3 \mathrm{MV} \mathrm{m}^{-1}$ for crystals $\mathrm{M}$ and $\mathrm{S}$ while $E_{180^{\circ}}^{c} \approx 0.04 \mathrm{MV} \mathrm{m}^{-1}$ for crystal C. The $E_{90^{\circ}}$. is around the same level of $E_{180^{\circ}}^{c}$ for each crystal. Notice that the coercive fields for crystals $\mathrm{M}$ and $\mathrm{S}$ are almost one order of magnitude larger than those for crystal C. Simulations of the actuation strains of these three crystals are shown in Fig. 4. It is evident that the developed framework is capable of explaining the wide differences in electrostrictive behaviors as shown in Fig. 3.

In summary, a framework is developed to predict the strain hysteresis of barium titanate single crystals under combined electric field and stress. It is shown that the depolarization and coercivities have a nontrivial effect on the attainable actuation strain.

The authors wish to thank K. Bhattacharya for many helpful suggestions and E. Burcsu for sharing the experimental data in Fig. 3. The authors are glad to acknowledge the partial supports under Grant Nos. 95-2221-E-002-166, 95-2221-E-002-116, and 95-EC-17-A-05-S1-017.

${ }^{1}$ S. E. Park and T. R. Shrout, J. Appl. Phys. 82, 1804 (1997).

${ }^{2}$ X. B. Ren, Nat. Mater. 3, 91 (2004).

${ }^{3}$ E. Burcsu, G. Ravichandran, and K. Bhattacharya, Appl. Phys. Lett. 77, 1698 (2000).

${ }^{4}$ E. Burcsu, G. Ravichandran, and K. Bhattacharya, J. Mech. Phys. Solids 52, 823 (2004).

${ }^{5}$ Y. C. Shu and K. Bhattacharya, Philos. Mag. B 81, 2021 (2001).

${ }^{6}$ Z. Li, C. M. Foster, X. H. Dai, X. Z. Xu, S. K. Chan, and D. J. Lam, J. Appl. Phys. 71, 4481 (1992).

${ }^{7}$ J. E. Huber, N. A. Fleck, C. M. Landis, and R. M. McMeeking, J. Mech. Phys. Solids 47, 1663 (1999).

${ }^{8}$ J. Shieh, J. E. Huber, and N. A. Fleck, Acta Mater. 51, 6123 (2003).

${ }^{9}$ Y. Su and G. J. Weng, Proc. R. Soc. London, Ser. A 462, 1573 (2006).

${ }^{10}$ D. Shilo, E. Burcsu, G. Ravichandran, and K. Bhattacharya, Int. J. Solids Struct. 44, 2053 (2007).

${ }^{11} \mathrm{~K}$. Bhattacharya, J. Y. Li, and Y. C. Shu (in preparation).

${ }^{12}$ G. Arlt and P. Sasko, J. Appl. Phys. 51, 4956 (1980).

${ }^{13}$ S. Wada, S. Suzuki, T. Noma, T. Suzuki, M. Osada, M. Kakihana, S. E. Park, L. E. Cross, and T. R. Shrout, Jpn. J. Appl. Phys., Part 1 38, 5505 (1999).

${ }^{14}$ J. Y. Li and D. Liu, J. Mech. Phys. Solids 52, 1719 (2004).

${ }^{15}$ D. Liu and J. Y. Li, Appl. Phys. Lett. 83, 1193 (2003).

${ }^{16}$ D. Liu and J. Y. Li, Appl. Phys. Lett. 84, 3930 (2004).

${ }^{17}$ F. Jona and G. Shirane, Ferroelectric Crystals (Pergamon, New York 1962). 